\title{
Existe relação entre o gênero do paciente e os desfechos do implante percutâneo de prótese valvar aórtica?
}

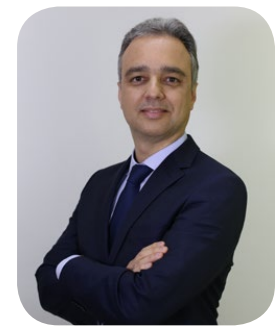

\author{
Adriano Dourado', Hilana Araújo¹, Roger Gonçalves Ribeiro¹
}

\begin{abstract}
Artigo Original: Gender-Related Differences on Short- and Long-Term Outcomes of Patients Undergoing Transcatheter Aortic Valve Implantation. Autores: Katz M, Nunes Filho ACB, Caixeta A, Carvalho LA, Sarmento-Leite R, Lemos Neto PA, São Thiago LEK, Oliveira ADD, Marino MA, Tumelero RT, Perin MA, Abizaid A, Tarasoutchi F, Brito FS. On behalf of the Brazilian TAVI Registry investigators. Catheter Cardiovasc Interv. 2017 Feb 15;89(3):429-436.
\end{abstract}

O implante percutâneo de prótese valvar aórtica (TAVI) é o procedimento de escolha para o tratamento da estenose aórtica calcificada em pacientes considerados inoperáveis ou de alto risco. ${ }^{1,2}$ Mais recentemente, com o resultado de importantes ensaios clínicos randomizados ${ }^{3,4}$, passou a ser considerado como alternativa à cirurgia convencional para pacientes de risco cirúrgico intermediário, desde que definida por equipe multidisciplinar (Heart Team). A ampliação das indicações estimulou a avaliação mais detalhada dos fatores de risco para complicações e insucesso do procedimento. No artigo, Katz e cols avaliaram a influência do sexo no risco de morte e complicações maiores em pacientes submetidos ao TAVI, com base nos dados do Registro Brasileiro para Avaliação dos Resultados do Implante por Cateter de Bioprótese Aórtica (RIBAC).

Foram avaliados os resultados de 809 pacientes, 418 do sexo feminino e 401 do sexo masculino, submetidos ao TAVI entre janeiro de 2008 e janeiro de 2015 , com média de seguimento de $497 \pm 478$ dias. As válvulas implantadas foram de primeira e segunda geração. $O$ grupo de pacientes do sexo feminino apresentava idade superior e maior alteração da função renal. Entretanto, as comorbidades foram mais prevalentes em pacientes do sexo masculino (Tabela 1). Os escores de risco cirúrgico, STS score e EUROscore não foram capazes de identificar as diferenças nos desfechos entre os dois grupos. Os principais achados foram: (1) maior incidência de complicações hemorrágicas graves ( $6 \%$ vs. $11,7 \% ; H R=0,50$ [95Cl\% 0,31-0,82]; $p=0.004)$ e vasculares importantes $(12,0 \%$ vs. $20,6 \%$; HR=0.57 [95\%Cl 0,40-0,81]; $\mathrm{p}=0,001$ ) no sexo feminino; (2) mortalidade em 30 dias significativamente superior entre as mulheres $e$ semelhante no seguimento tardio (Figura 1). Utilizando modelo de regressão logística de Cox foram identificados fatores de risco independentes para morte de qualquer causa: diabetes mellitus, doença arterial periférica (DAP), função renal e doença pulmonar obstrutiva crônica (DPOC). Quando separados por sexo, mantiveram-se como fatores independentes de morte a DPOC e o diabetes para homens, e a DAP e função renal para mulheres. Não houve diferença na ocorrência de eventos cardiovasculares maiores (MACE) combinados. Esses achados, em uma amostra da população brasileira, são semelhantes aos de outros registros com diferentes populações.

As complicações hemorrágicas da TAVI são, na maioria das vezes, decorrentes de complicações vasculares. O maior perfil das próteses de primeira e segunda geração associado a vasos de menor calibre em mulheres, apesar de não ter sido avaliada nessa publicação, pode ter colaborado para esse resultado. ${ }^{5}$ Outro dado relevante foi a prevalência maior de características clínicas associadas a maior sangramento, como idade avançada, baixo peso, doença aterosclerótica coronariana e disfunção renal, no grupo de sexo feminino.

De modo geral, a mortalidade em 30 dias está diretamente relacionada ao procedimento a que é submetido o paciente e às suas complicações, justificando assim esse desfecho mais frequente em mulheres submetidas à TAVI. Por outro lado, a mortalidade no seguimento a médio e longo prazo é superior no sexo masculino e está associada à presença de comorbidades. Katz e cols não encontraram diferença na mortalidade no seguimento total do estudo a despeito da prevalência maior de comorbidades em homens. É provável que a maior ocorrência de complicações e morte relacionada ao procedimento foram minimizadas pela maior sobrevida das mulheres no seguimento tardio.

Em recente estudo observacional com dispositivo de $3^{\text {a }}$ geração, Sapien 3, Szerlip e cols não encontraram diferenças na mortalidade em 30 dias entre ho- 
mens e mulheres. ${ }^{6}$ Apesar de uma maior ocorrência de complicações vasculares no sexo feminino, não houve diferença significativa de complicações hemorrágicas. Interessante notar que a incidência de complicações vasculares maiores em mulheres foi $5 \%(7,2 \%$ vs. $11,2 \%)$, menor do que no estudo brasileiro.

$O$ artigo traz valiosas informações sobre o TAVI na população brasileira, demonstrando resultados seme-
Ihantes aos de outros grupos. O sexo feminino aparece como fator de risco para complicações vasculares e hemorrágicas, com consequente aumento na mortalidade relacionada ao procedimento. Não obstante, é preciso relativizar os resultados com base no grupo de pacientes estudados - média de idade $>80$ anos e risco cirúrgico proibitivo - e o uso de dispositivos de primeira e segunda geração, já não mais em uso.

Tabela 1 - Características clínicas de acordo com o gênero.

\begin{tabular}{|c|c|c|c|c|}
\hline & Women $(N=418)$ & $\operatorname{Men}(N=401)$ & Total $(N=819)$ & $P$-value \\
\hline Age (years) & $82.4 \pm 7.0$ & $80.6 \pm 7.5$ & $81.5 \pm 7.3$ & $<0.001$ \\
\hline Body weight (kg) & $65.8 \pm 14.4$ & $75.2 \pm 13.7$ & $70.4 \pm 14.8$ & $<0.001$ \\
\hline BMI $\left(\mathrm{kg} / \mathrm{m}^{2}\right)$ & $26.6 \pm 5.4$ & $26.0 \pm 4.0$ & $26.3 \pm 4.7$ & 0.09 \\
\hline NYHA class III or IV, $n$ (\%) & $354(85)$ & $312(78)$ & $666(81)$ & 0.01 \\
\hline LVEF $(\%)$ & $61.9 \pm 13.4$ & $55.4 \pm 15.6$ & $58.7 \pm 14.9$ & $<0.001$ \\
\hline $\operatorname{AVA}\left(\mathrm{cm}^{2}\right)$ & $0.6 \pm 0.2$ & $0.7 \pm 0.2$ & $0.7 \pm 0.2$ & $<0.001$ \\
\hline Mean aortic gradient (mm $\mathrm{Hg}$ ) & $52.2 \pm 15.7$ & $46.3 \pm 15.7$ & $49.3 \pm 16.0$ & $<0.001$ \\
\hline Hypertension, $n(\%)$ & $323(77)$ & $294(73)$ & $617(75)$ & 0.19 \\
\hline Diabetes mellitus, $n$ (\%) & $114(27)$ & $144(36)$ & $258(31)$ & 0.008 \\
\hline COPD, $n(\%)$ & $65(16)$ & $89(22.0)$ & $154(19)$ & 0.015 \\
\hline PVD, $n(\%)$ & $61(15)$ & $79(20)$ & $140(17)$ & 0.052 \\
\hline $\mathrm{CAD}, n(\%)$ & $197(47)$ & $283(71)$ & $480(59)$ & $<0.001$ \\
\hline Previous MI, $n$ (\%) & $41(10)$ & 78 (19) & 119 (14) & $<0.001$ \\
\hline Previous stroke, $n$ (\%) & $26(6)$ & $39(10)$ & $65(8)$ & 0.064 \\
\hline STS score (\%) & $15.4 \pm 13.0$ & $11.4 \pm 9.5$ & $13.5 \pm 11.6$ & $<0.001$ \\
\hline Logistic EuroSCORE (\%) & $20.4 \pm 14.7$ & $20.9 \pm 16.4$ & $20.6 \pm 15.6$ & 0.63 \\
\hline Hemoglobin (mg/dL) & $11.4 \pm 1.5$ & $12.1 \pm 1.9$ & $11.8 \pm 1.8$ & $<0.001$ \\
\hline AAS, $n(\%)$ & $403(96)$ & $389(97)$ & $792(97)$ & 0.63 \\
\hline Clopidogrel, $n$ (\%) & $389(93)$ & $384(96)$ & $773(94)$ & 0.09 \\
\hline Pulmonary hypertension, $n$ (\%) & $90(21)$ & $89(22)$ & $179(22)$ & 0.82 \\
\hline $\mathrm{CrCl}<60 \mathrm{ml} / \mathrm{min}, n(\%)$ & $335(80)$ & $278(69)$ & $613(75)$ & $<0.001$ \\
\hline Creatinine clearance $(\mathrm{ml} / \mathrm{min})$ & $45.8 \pm 20.6$ & $51.1 \pm 23.1$ & $48.4 \pm 22.0$ & $<0.001$ \\
\hline
\end{tabular}

BMI, body mass index; NYHA, New York Heart Association; LVEF, left ventricle ejection fraction; AVA, aortic valve area; COPD, chronic obstructive pulmonary disease; PVD, peripheral vascular disease; $\mathrm{CAD}$, coronary artery disease; $\mathrm{MI}$, myocardial infarction; $\mathrm{CrCl}$, creatinine clearance.

Figura 1 - Curva de sobrevida de acordo com o gênero.

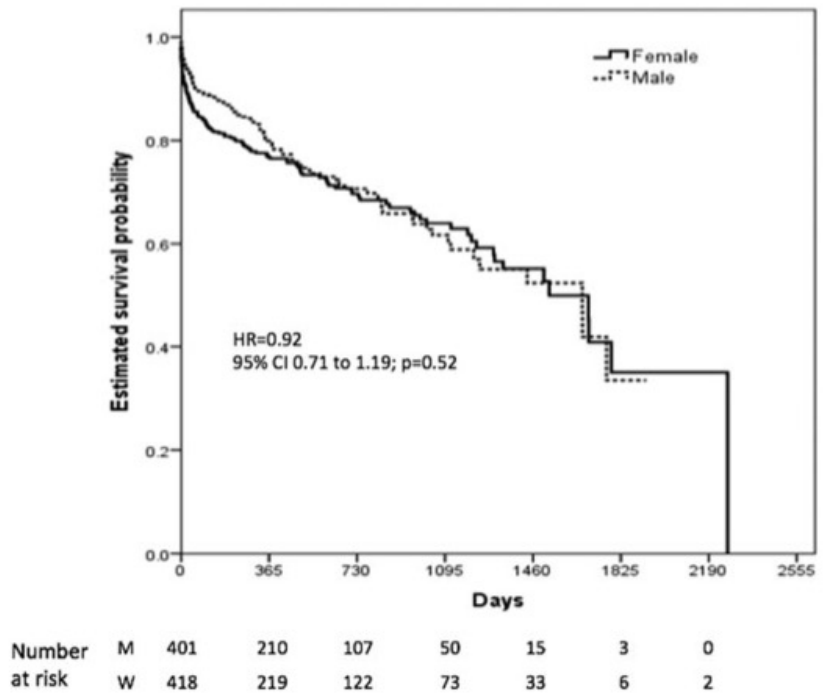




\section{REFERÊNCIAS}

1. Nishimura RA, Otto CM, Bonow RO, Carabello BA, Erwin JP 3rd, Fleisher LA, Jneid H, Mack MJ, McLeod CJ, O'Gara PT, Rigolin VH, Sundt TM 3rd, Thompson A. 2017 AHA/ACC focused update of the 2014 AHA/ACC guideline for the management of patients with valvular heart disease: a report of the American College of Cardiology/American Heart Association Task Force on Clinical Practice Guidelines. Circulation. 2017;135:e1159e1195.DOI:10.1161/CIR.0000000000000503.

2. Baumgartner $H$, Falk $V$, Bax JJ, De Bonis $M$, Hamm C, Holm PJ, lung B, Lancellotti P, LansacE, Rodriguez Muñoz D, Rosenhek R, Sjögren J, Tornos Mas P, Vahanian A, Walther T, Wendler O, Windecker S, Zamorano JL; ESC Scientific Document Group.2017 ESC/ EACTS Guidelines for the management of valvular heart disease.European Heart Journal, Volume 38, Issue 36, 21 September 2017, Pages 2739-2791, https://doi. org/10.1093/eurheartj/ehx391.

3. Leon MB, Smith CR, Mack MJ, Makkar RR, Svensson LG, Kodali SK, Thourani VH, Tuzcu EM, Miller DC, Herrmann HC, Doshi D, Cohen DJ, Pichard AD, Kapadia S, Dewey T, Babaliaros V, Szeto WY, Williams MR, Kereiakes D, Zajarias A, Greason KL, Whisenant BK, Hodson RW, Moses JW, Trento A, Brown DL, Fearon WF, Pibarot P, Hahn RT, Jaber WA, Anderson WN, Alu MC, Webb JG; PARTNER 2 Investigators. Transcatheter or Surgical Aortic-Valve Replacement in Intermediate-Risk Patients. N Engl J Med. 2016 Apr 28;374(17):1609-20. doi: 10.1056/NEJMoa1514616. Epub 2016 Apr 2.

4. Reardon MJ, Van Mieghem NM, Popma JJ, Kleiman NS, Søndergaard L, Mumtaz M, Adams DH, Deeb GM, Maini B, Gada H, Chetcuti S, Gleason T, Heiser J, Lange R, Merhi W, Oh JK, Olsen PS, Piazza N, Williams M, Windecker S, Yakubov SJ, Grube E, Makkar R, Lee JS, Conte J, Vang E, Nguyen H, Chang Y, Mugglin AS, Serruys PW, Kappetein AP; SURTAVI Investigators. Surgical or Transcatheter Aortic-Valve Replacement in Intermediate-Risk Patients. N Engl J Med. 2017 Apr 6;376(14):1321-1331. doi: 10.1056/NEJMoa1700456. Epub 2017 Mar 17.

5. Azarbaijani Y1, O'Callaghan K, Sanders WE, Wu C, Laschinger J, Marinac-Dabic D, Strauss DG, Canos DA, Zusterzeel R.Sex-Specific Outcomes After Transcatheter Aortic Valve Replacement: A Review of the Literature.Cardiol Rev. 2018 Mar/Apr;26(2):73-81. doi: 10.1097/CRD.0000000000000177.

6. Szerlip et. al. Sex-Specific Outcomes of Transcatheter Aortic Valve Replacement with the Sapien 3 Valve. JACC Intv 2018;11:13-20. doi.org/10.1016/j. jcin.2017.09.035.
1. Serviço de Cardiologia Intervencionista do HSI Endereço para correspondência: addoliveira@hotmail.com 